\title{
¿Por qué es tan difícil legislar sobre alcohol en España?
}

\author{
Alicia Rodríguez-Martos ${ }^{1,2}$ \\ ${ }^{1}$ Agència de Salut Pública de Barcelona. \\ ${ }^{2}$ CIBER Epidemiología y Salud Pública (CIBERESP), España.
}

Enviar correspondencia a:

Alicia Rodríguez-Martos. Agència de Salut Pública de Barcelona. PI Lesseps, 1 Barcelona 08023. Tel.: 3493 202 7705. E-mail: amartos@aspb.cat

\section{RESUMEN}

El vino forma parte de la cultura mediterránea. Beber alcohol es parte de nuestra vida social y sus consecuencias adversas a menudo se han minimizado, culpando más bien al individuo, que no sabe beber, que al agente causante del daño.

En los últimos años, los accidentes de tráfico y el consumo 'en atracones' de los jóvenes han logrado poner el alcohol en la agenda política. En efecto, los adolescentes han incrementado su consumo de alcohol y desarrollado una pauta de episodios de consumo intensivo, insertado en el ocio nocturno en espacios abiertos, donde masas de jóvenes beben al aire libre botellas grandes ('botellón').

Se han generado campañas y materiales educativos, pero dos sucesivos anteproyectos de ley sobre medidas de control para prevenir el daño relacionado con el alcohol han fracasado en su intento de ser aprobadas, al chocar dos veces con los mismos intereses creados.

El primer anteproyecto (2002) se orientaba a la prevención de problemas de alcohol en la población general. El segundo (2006), se limitaba a la prevención en menores. Nadie, ni siquiera la industria, podía estar en contra. Pero un sesgado interés puso el acento en el vino - que, presuntamente, no debería ser considerado alcohol, sino alimento y, en consecuencia, debía eliminarse de la ley - de modo que el anteproyecto se convirtió en la 'guerra del vino', con sus predecibles consecuencias. De nuevo, un anteproyecto de ley sobre alcohol se guardaba sine die en el 'baúl de los recuerdos'.

Dentro de este panorama pesimista, todavía existe algún indicio positivo. Ninguna razón económica ni política debería obstaculizar siquiera la protección de los menores.

Palabras clave: politica de alcohol, medidas de control, jóvenes.

\section{ABSTRACT}

Wine belongs to the Mediterranean culture. Drinking alcohol is part of our social life, and its negative consequences have often been minimised, blame being apportioned rather to the individual, who doesn't know how to drink, than to the agent causing the harm.

In recent years, road accidents and binge-drinking among young people have put alcohol on the political agenda. Adolescents and young adults have increased their consumption, and a pattern of binge-drinking has developed based on the open-air 'fiesta' nightlife, where groups of young people drink large bottles in the streets and squares (botellón).

Educational campaigns and material have been mounted and produced, but two consecutive draft laws on measures for the prevention of alcohol-related harm have failed to be approved, coming up on both occasions against the same vested interests.

This editorial reviews the ambivalence of society towards regulations on alcohol and the current state of the art.

The first draft law (2002) was about preventing alcohol problems in the general population; the second (2006) was limited to the question of prevention among minors. Nobody could oppose it, not even the industry. However, an interested bias put the accent on wine - which allegedly should be considered not as alcohol but rather as food, and consequently removed from the law - so that discussion of the draft law became a 'wine war', with predictable consequences. Once again, a draft law on alcohol was shelved sine die and tossed into a corner to gather dust.

Within this pessimistic scene, though, there are still some positive signs. No economic or political motives should constitute an obstacle to the protection of minors, at the very least.

Key words: alcohol policy, control measures, young people. 


\section{INTRODUCCIÓN}

E spaña, como país mediterráneo, tiene hondas raíces vitivinícolas. La mitología, la tradición mediterránea y el saber popular explican la consolidación de una cultura alcohólica en la que los excesos han sido banalizados, en tanto sus consecuencias han podido ser contenidas, o denostados, cuando éstas han trascendido o el bebedor ha quedado atrapado en la dependencia. La tendencia espontánea por parte de la población ha sido culpar al sujeto de 'no saber beber', olvidando el poder adictivo de la sustancia. Es decir, cualquier consecuencia adversa del consumo de alcohol, ha sido con frecuencia imputada al sujeto y no al agente, ignorando o infravalorando además, con frecuencia, el entorno favorecedor de los excesos y el consumo de riesgo. Se busca un chivo expiatorio (ahora, los jóvenes) y se olvida la sociedad que fomenta su luna de miel con el alcohol.

Esta visión simplista es fomentada por la industria alcoholera y otros sectores -directa o indirectamentebeneficiados por el negocio de las bebidas alcohólicas (industria agrícola, productores de alcohol, distribuidores y vendedores, entre los cuales cabe destacar la poderosa industria del ocio, especialmente el nocturno), ambos beneficiarios de la generalización del consumo de alcohol asociado al uso del ocio y estilos de vida juveniles hoy en boga, como el 'ir de marcha', cuyo eje vertebrador parece ser el consumo de alcohol. Los menores de edad, que cada vez se suman antes a la 'tribu' marchosa, quedan atrapados en esta misma dinámica y constituyen, de hecho, el núcleo principal de los 'botelloneros'.

Sin embargo, el creciente consumo de bebidas alcohólicas por parte de los jóvenes ${ }^{1}$ y las molestias inherentes al 'botellón', junto con las muertes y lesiones de tráfico atribuibles al etanol', contribuyeron a poner el alcohol en la agenda política.

En los últimos años, se han lanzado campañas divulgativas y producido materiales informativos. El botellón, piedra de escándalo para la mayoría de la sociedad adulta, se ha convertido en un elemento al mismo tiempo perturbador del orden social (molestias vecinales) e integrador (cohesión social entre pares). Sin embargo, los dos intentos de legislar sobre estrategias preventivas han fracasado, al tropezar con numerosas barreras, donde coinciden presuntas ideologías progresistas, poco amantes de las leyes, e intereses creados del sector económico. Determinadas circunstancias, como la recientemente vivida con el anteproyecto de ley sobre alcohol promovido por la ex-ministra Salgado, ponen de manifiesto flagrantes contradicciones y la triste realidad de que el conflicto entre economía y salud se salda siempre a favor de la primera.

\section{INICIATIVAS DE REGULACIÓN}

E el año 2002, el gobierno del Partido Popular presentó un Anteproyecto de ley de prevención del abuso de alcohol, que, aunque bautizada con el mote de la 'ley del botellón', se refería a toda la población y abarcaba una serie de medidas de control del consumo, que el Consejo Económico y Social consideró excesivamente sancionadoras y perjudiciales para el sector productor, proponiendo en cambio que se excluyeran de la aplicación de la ley las bebidas naturales de carácter agrícola y uso alimentario que dispongan de características específicas ${ }^{2}$. El anteproyecto se estrelló contra el sólido dique del sector alcoholero, que en cambio logró que se aprobara la Ley 24/2003, de 10 de julio, de la Viña y el Vino, donde se define el vino como alimento. Una buena medida, presuntamente protectora frente a posibles futuros intentos de una nueva ley sobre el alcohol, donde, a tenor de esta definición del vino, éste debería ser excluido de las bebidas alcohólicas.

Entretanto, nuestros jóvenes siguen instalados en el 'botellón', versión ibérica del patrón anglosajón - hoy imperante en los jóvenes europeos, de episodios de consumo intensivo de alcohol, concentrado en pocas horas- y lo han llevado a los espacios abiertos, donde consumen los suministros comprados en el supermercado. Ante este fenómeno en expansión, desbordado en el año 2006 con las convocatorias de 'macrobotellones' a través de internet y móviles, que pugnaban entre sí por batir el record de participación, la sociedad responde dividida. Por un lado, los padres sufren por los peligros en que incurren sus hijos, aunque a menudo se infravalora el riesgo para su salud presente y futura ante al peligro más inmediato del accidente de tráfico. Por otro, el vecindario se queja de las molestias que comporta el 'botellón' (ruido, vandalismo, vómitos, orines...). En cuanto a los responsables políticos, algunas autoridades locales se conforman con que los chavales no molesten a los vecinos, y les buscan espacios apartados e incluso especialmente habilitados para hacer 'botellón' ('boteIlódromos', en Andalucía), o no conduzcan bebidos (trasporte público especial o con horario ampliado; campañas de 'conductor designado'), mientras otros se preocupan además por el fondo de la cuestión: esta forma de divertirse de muchos jóvenes, cuyo ocio nocturno suele consistir en 'ir de marcha' bien 'colocadito', con lo que ello presupone de riesgo para su salud y, a menudo, de empobrecimiento personal.

El amplio eco mediático del 'botellón' y del elevado consumo de alcohol de los jóvenes, junto a la consiguiente alarma social, por un lado, y la recién impulsada Estrategia Europea sobre $\mathrm{Alcohol}^{3}$, por el otro, hacían el momento idóneo para retomar el fallido anteproyecto de alcohol, limitándolo en esta ocasión a la protección de los menores, algo en lo 
que todos los agentes sociales, incluso la industria, parecían coincidir.

Sin embargo, el nuevo Anteproyecto de la Ley de medidas sanitarias para la protección de la salud y la prevención del consumo de bebidas alcohólicas por menores (25-09-2006; enmendado el 12-2-2007; suspendida su tramitación el 20-2-2007), presentado esta vez por el gobierno socialista, volvió a estrellarse contra los sectores implicados, desde productores hasta el sector publicitario y sus beneficiarios, y muy especialmente contra el sector del vino, que vaticinó un apocalipsis para la industria vitivinícola española. El Anteproyecto, caricaturizado como 'cruzada de la ley seca', se encontró por el otro lado con la cruzada del honor patrio.

Aunque el Anteproyecto se refería a limitaciones de venta, dispensación y consumo de bebidas con un contenido alcohólico superior al 1,2\% por parte de los menores de 18 años, así como a la publicidad, promoción y patrocinio dirigida a ese mismo sector poblacional, se pretendió - de forma interesada- identificar al Anteproyecto como una guerra contra el vino y como un atentado a la libertad de expresión publicitaria. De hecho, fueron la inclusión del vino como bebida alcohólica y las limitaciones a la publicidad los aspectos que generaron mayor encono en esta guerra no declarada. Se posicionaron en contra la industria agrícola y alcoholera, en especial el sector del vino y algunas Comunidades Autónomas, algunas de las cuales, como Castilla-La Mancha (importante productora de alcohol), pusieron el grito en el cielo ante la anunciada 'ley seca' que pretendía implantar un ministerio 'prohibicionista' cuando, en realidad tenían ya una normativa, análoga a la propuesta, aprobada en su propio territorio. Para algunos, el vino, una bebida obtenida por fermentación y con graduación superior a los $10^{\circ}$ de etanol, no es alcohol, sino un alimento integrante de la beneficiosa 'dieta mediterránea'. Desde la patronal del vino se dijo que, aún estando de acuerdo en que los menores no debían consumir bebidas alcohólicas, había que enseñarles a beber vino para que supieran hacerlo 'responsablemente' a partir de los 18 años. Las cortapisas a la publicidad fueron rechazadas tanto por la industria alcoholera como por los publicistas y los medios que se financian con ella.

Querer controlar el acceso de los menores al alcohol (vino incluido), hizo rasgarse las vestiduras también a políticos e intelectuales y, como no, a la FAS (Fundación Alcohol y Sociedad, fundada por FEBE, Federación Española de Bebidas Espirituosas), presuntamente creada para prevenir las consecuencias adversas del consumo de alcohol, especialmente por parte de los menores y jóvenes. Uno de los miembros de su comité científico, José Antonio Marina, respetado filósofo -no experto en adicciones, que se sepa-, consideraba una cuestión ética el abuso de sustancias y el consumidor 'abusivo' como un ser merecedor de rechazo social. Defendía, en consecuencia, como todo el comité científico de la FAS, educar para un consumo responsable. (El Mundo, 4-02-07). La industria alcoholera y sus ad lateres, como Fundación Alcohol y Sociedad, han apostado por la prevención y ofrecen incluso programas de educación en la escuela, además de vistosas campañas como la del 'Conductor Alternativo', en la que premian la sobriedad del conductor alternativo con mucho aparato mediático, mientras se oponen, en cambio, a una regulación legal de la publicidad y a cualquier otra medida probadamente eficaz como el aumento de impuestos y precios. La actitud de concentrar toda la culpa en el individuo, exonerando al alcohol, goza de muchos adeptos. El secreto está en saber beber de forma responsable. El discurso del individuo 'responsable' ha cautivado al gran público, porque no hay que hacer nada ${ }^{4}$. De entrada, nadie se da por aludido, ya que la mayoría de bebedores consideran que 'controlan' y, para los políticos, pasarle la responsabilidad al individuo es la mejor forma de evitar meterse 'en camisa de once varas'.

También se declararon en contra del reciente Anteproyecto políticos del Partido Popular, impulsor del frustrado Anteproyecto anterior, y la Ministra de Agricultura del actual gobierno socialista, cofrade del vino.

Las opiniones a favor del Anteproyecto de la mayoría de sociedades médicas (32, en concreto), de padres de alumnos y organizaciones de consumidores fueron recogidas en un Manifiesto (7-02-07), que subrayaba los efectos perjudiciales del alcohol sobre el cerebro adolescente, apoyaba la prohibición y venta de alcohol a los menores y reclamaba políticas de restricción de la publicidad. El manifiesto fue duramente criticado por actuar por intereses aviesos (i?).

Finalmente, 'por Santiago y por España', se suspendió de forma indefinida la tramitación del Anteproyecto. Mientras los sanitarios todavía lamentamos la oportunidad perdida, el presidente de la Federación Española del Vino celebraba la retirada del anteproyecto, aunque temía que Sanidad volvería a la carga: ("Hemos ganado una batalla, pero me temo que la guerra va a continuar." El Mundo, 18-06-07), manteniendo la actitud victimista y sesgada del sector, todavía sostenida por personas de relevancia política, como el ex ministro Pimentel que, reflexionando sobre el momento del vino español, aludía al abortado proyecto de ley, que lo 'penalizaba'(Cinco Días, 25-07-07). Y, como si se tratara de un chiste, aunque paradigmático de nuestras patrióticas contradicciones, el victimizado sector del vino, concretamente de la Fundación Castilla-La Mancha, Tierra de Viñedos, lanzaba una campaña, en Junio de 2007, según la cual "sabemos hacer buen vino, sin rebasar los límites permitidos" y se pueden tomar dos copas de vino sin que las detecte el alcoholímetro, a lo que respondió la 
Dirección General de Tráfico (DGT) con una multa de 30.000€ (El Periódico, 24-06-07).

A todo esto, los padres, cuya colaboración y responsabilidad respecto al control de sus hijos invocaba la ministra, siguen pidiéndole al gobierno que haga algo y Rodrigo Córdoba, portavoz del Comité Nacional de Prevención del Tabaquismo, se preguntaba, en una carta al director (El País, 23-02-07), si la salud pública perjudica a los políticos.

Es ciertamente lamentable ver paralizado un anteproyecto de ley tan necesario y urgente como el que acaba de bloquearse. Las reclamaciones de las familias y de los profesionales, conscientes del perjuicio inmediato (intoxicaciones agudas de menor o mayor gravedad, lesiones) o demorado (afectación cerebral, adicción) que el alcohol representa para los adolescentes, ya sea en forma de cerveza, vino o destilados, no han servido para apoyar a las autoridades sanitarias en su loable empeño de proteger a los menores, sino que todos han fracasado estrepitosamente frente a intereses económicos y políticos. La protección de la salud de nuestros menores, nuestro bien más preciado y nuestro futuro, es inexcusable e indemorable y debería estar por encima de cualquier conflicto de intereses.

\section{¿ES POSIBLE PREVENIR SIN CONTROLAR LA EXPOSICIÓN AMBIENTAL?}

Entre los factores de riesgo para desarrollar problemas de alcohol, además de los individuales tales como la predisposición genética, los trastornos de personalidad, la falta de atención y/o los límites, normas y ejemplo por parte de los padres, etc., existen factores del entorno social. La buena imagen del alcohol y el acceso fácil a las bebidas alcohólicas a un precio asequible, así como el microclima social donde el joven se desarrolla, juegan un papel indudable. Las pautas de consumo de los iguales, y el modelado social que se plasma en los medios de comunicación, van calando hondo y configurando la imagen interna de lo que debe ser la diversión de un joven de hoy. $Y$ la imagen que se vende es la de la diversión asociada a los consumos de drogas, donde el alcohol es el eje omnipresente. El alcohol se asocia a 'la marcha', al ocio nocturno, al éxito social y sexual, al glamour y al estilo más rompedor, incluso al deporte. Beber alcohol es divertido. Los no bebedores son sosos y menos populares. La imagen que se da de forma reiterada a través de la publicidad y de las series televisivas fomenta la intención de la conducta de beber, por lo que, incluso el joven que no es directamente presionado, siente que se espera de él que beba y, mayoritariamente, lo acaba haciendo. La edad, la medida y forma en que se materialice el consumo dependerá de las circunstancias. Las campañas publicitarias sue- len estar mejor diseñadas que las preventivas porque parten de finos análisis sobre los objetivos, el grupo diana y sus motivaciones, así como sobre el tipo de mensaje de mayor impacto.

Se estima ${ }^{5}$ que los medios de comunicación tienen un papel fundamental "en la adquisición de conocimientos, valores / actitudes y pautas de comportamiento a través de la producción de sentido (making sense)", para lo bueno y para lo malo. La regulación de la publicidad queda recogida en España en la Ley 34/1988, General de Publicidad, y en la Ley 22/99, que incorpora la Directiva 89/552/CEE, más conocida como Directiva de la Televisión sin Fronteras. La Ley 34/1988 ya obliga a la clara identificación de los mensajes publicitarios y a su veracidad, prohibiendo tanto la publicidad subliminal como la engañosa, con lo que podía interpretarse como ilícito el emplazamiento del producto o product placement.

En cuanto al contenido de los anuncios tanto nuestra legislación como la europea, incluidos los códigos de autorregulación, prohíben explícitamente ciertas asociaciones con el alcohol - éxito social, deportivo, sexual, etc.-, así como la aparición de menores o que el mensaje esté directamente dirigido a ellos. Tampoco puede ridiculizarse la abstinencia o incitar al consumo desmedido.

Los menores son grandes y crecientes consumidores de televisión, siendo la tarde la hora de mayor audiencia, aunque es muy frecuente que la vean por la noche, sobre todo en torno a la hora de cenar. Pues bien, muchas series de sobremesa incluyen consumos cuantiosos de alcohol, con emplazamiento de las marcas (especialmente cervezas) y a menudo protagonizados por jóvenes en el límite de la edad legal. El emplazamiento del producto, hasta ahora objeto de controversia acerca de su legalidad, acaba de ser regulado en la Directiva 89/552/CEE Televisión sin Fronteras, enmendada de forma que se autoriza, siempre y cuando se haga constar al principio del programa, que se inserte publicidad del producto. Esta especificación excluye el product placement de la publicidad ilícita o subliminal y acaso sea una solución razonable para una marca de detergente, pero constituye una vía para salvar otras limitaciones, en el caso del alcohol y para poder penetrar mucho más sutilmente (a través de la acción dramática y la normalización de conductas modelo). En efecto, la Directiva modificada regula el emplazamiento del producto y lo prohíbe en programas informativos e infantiles y en relación con determinados productos, como el tabaco, pero se olvida del alcohol. Nuestra Ley 34 / 1988 General de Publicidad sólo prohíbe la publicidad de las bebidas alcohólicas con un contenido en etanol superior a $20^{\circ}$, lo que no impide que tanto los espacios publicitarios como los programas estén inundados de cerveza, insertada en la acción (emplazamiento del producto) o en forma de patrocinios. 


\section{¿Y SE PUEDE CONTROLAR LA OFERTA SIN LEGISLAR?}

Prevenir o reducir el daño relacionado con el consumo de bebidas alcohólicas sin controlar la disponibilidad de las mismas es como querer llenar una pica de agua sin poner un tapón en el desagüe. El informe de Babor et al, $2003^{6}$ mostró sin lugar a dudas que las estrategias preventivas eficaces y rentables eran especialmente las que limitaban la disponibilidad y promoción de las bebidas alcohólicas y elevaban su precio. El referido informe recuerda que cualquier política pública consiste en decisiones autoritarias tomadas por gobiernos, debiendo ser éstos y no la industria quienes deciden; asimismo define la política de alcohol como aquella que se ocupa de la relación entre alcohol, salud y bienestar social.

La literatura científica ${ }^{6}$ nos indica que la educación escolar es muy poco eficaz como instrumento preventivo. La educación familiar (comunicación verbal y no verbal), es más poderosa de lo que los padres creen y los programas preventivos basados en el refuerzo de la comunicación familiar, son los más efectivos. Sin embargo, la prevención educativa debe ser necesariamente complementada por medidas comunitarias del control de la oferta, sirviendo la educación familiar y escolar más bien como base para facilitar la aceptación y sostenibilidad de otras medidas de eficacia preventiva probada.

Aunque los españoles tenemos un dicho que reza 'hecha la ley, hecha la trampa', sin legislación no se puede controlar la disponibilidad del alcohol por parte de los menores ni su promoción comercial. Tampoco otras medidas de control más generales, como las destinadas a reducir la accidentalidad relacionada con el alcohol al volante. Cualquier cambio de conducta en el conductor deriva en primer lugar de controles externos, (velocidad, alcoholemia, etc.), a los que sucede un control social (conductas socialmente reprobadas) y finalmente un control interno (la convicción con que se rechazan y cambian ciertos hábitos), el control más importante, pero el que más tarda en desarrollarse. Estos factores psicológicos, implicados en la efectividad de las estrategias preventivas ${ }^{7}$ en general, son coherentes con la imperiosa necesidad de legislar, además de educar a la sociedad y al individuo.

Los gobiernos, y en concreto las autoridades sanitarias, tienen el deber de velar por nuestra salud y eso no es el 'integrismo de la promoción de salud', como ha dicho alguno, ni un atentado contra la libertad individual.
El último anteproyecto retirado se refería a menores y era coherente con las Conclusiones del Consejo de Europa ${ }^{8}$ de 5 de junio de 2001 sobre una estrategia comunitaria para reducir los daños derivados del consumo de alcohol, y con la consiguiente Recomendación del Consejo de Ministros de Sanidad de 5 de junio de $2001^{9}$ sobre el consumo de alcohol por parte de los jóvenes y en particular a niños y adolescentes, según la cual los Estados miembro deben elaborar estrategias preventivas y acciones reguladoras destinadas a proteger a menores y jóvenes con políticas tanto globales como específicas de promoción de la salud en este colectivo.

En respuesta a la mencionada Recomendación ${ }^{9}$, la Dirección General de Salud y Protección del Consumidor de la Comunidad Europea propuso, el pasado mes de octubre, una Estrategia de Alcohol ${ }^{3}$. Una vez aceptada y recomendada por el Consejo de Europa, en la actualidad se están desarrollando los mecanismos y estructuras necesarios para su implementación y seguimiento. Es de subrayar que, entre los 5 ítems prioritarios que propone la Estrategia, el primero es precisamente la protección de jóvenes, niños y neonatos.

La propuesta de un Anteproyecto de ley para proteger a los menores de los daños relacionados con el consumo de alcohol no podía, pues, darse en momento más oportuno, tanto por la necesidad urgente a nivel nacional como por la idoneidad de seguir el ritmo que se intenta llevar desde la Comunidad Europea.

A pesar de que casi la totalidad de comunidades autónomas españolas tienen normas reguladoras del consumo de alcohol por parte de menores (edad legal* para la adquisición de bebidas alcohólicas y prohibiciones o limitaciones a la venta y promoción), poca es la legislación a nivel estatal sobre alcohol, excepción hecha de la prohibición de vender alcohol por debajo de los 16 años en establecimientos, espectáculos y actividades recreativas y de la prohibición de la publicidad de bebidas de contenido alcohólico superior a $20^{\circ}$ por televisión.

No es, pues, de extrañar, que el retirado Anteproyecto de ley español pusiera el acento en la edad legal para comprar y consumir alcohol (18 años), así como en otras limitaciones para la dispensación como ciertos lugares y horas y en las restricciones a la promoción de las bebidas alcohólicas a fin de que la población menor quedara menos expuesta a los mensajes publicitarios.

Otra estrategia preventiva impulsada por la UE en el año 2006, de carácter menor pero a la que no se debería renunciar, es el etiquetado de los envases

\footnotetext{
* A nivel estatal, aún es de 16 años, aunque la inmensa mayoría de CCAA ha subido la edad legal a 18 años, tal y como recomienda la Comisión Europea.
} 
de bebidas alcohólicas, incluyendo su graduación, el número de unidades de bebida contenido en la botella y alguna información básica relativa a la importancia de no consumir por parte de embarazadas y conductores.

En el pasado mes de junio, la ministra Salgado anunció que no se retomaría la tramitación de la ley de alcohol en esta legislatura, aunque la retirada se consideraba "indefinida, pero no permanente". Sin embargo, cabe celebrar la firma de un par de convenios, retomando algunos de los aspectos del interrumpido anteproyecto.

El día 11, se firmaba un Convenio de Colaboración entre el Ministerio de Sanidad y Consumo y la Asociación de Editores de Diarios (AEDE), entidad que incluye 93 medios, en virtud del cual los editores elaborarán un código de autorregulación. El convenio, establecido por un año prorrogable, será objeto de seguimiento por parte de una comisión mixta. Entre sus cláusulas, se establece la elaboración de un código de autorregulación, que deberá incluir el compromiso de no insertar publicidad de bebidas alcohólicas en ninguna publicación o sector específicamente dirigida a menores de 18 años; no insertar publicidad en las cubiertas exteriores, portada y contraportada, además de no insertar anuncios que incumplan con la normativa ya vigente en cuanto a contenidos (no aparición de menores, no asociaciones del consumo con éxitos diversos, promoción el consumo 'inmoderado', etc). El convenio compromete a los editores de diarios a colaborar en las campañas divulgativas del MSC, la entrega gratuita de un encarte o folleto informativo sobre los efectos del consumo de alcohol en menores, así como a promover, entre sus asociados, la inclusión de una advertencia sanitaria referida al daño que el consumo de bebidas alcohólicas puede producir en menores junto a cada anuncio de bebida alcohólica.

Confiemos en que este convenio se cumpla y renueve anualmente. La Asociación de Usuarios de la Comunicación $(A \cup C)^{10}$ ha pedido un convenio similar para las revistas y la radio, e instado a contar con padres y consumidores para el código deontológico propuesto. Sería altamente deseable esta extensión, habida cuenta de la profusa publicidad que se realiza desde esos medios no incluidos, especialmente desde la radio, nunca legislada y que no sólo inserta anuncios, sino patrocinios que generan múltiples mensajes a menudo mezclados con el propio programa por el locutor.

El 13 de junio, se firmó otro Convenio de colaboración entre el Ministerio de Sanidad y Consumo y ANGED (patronal de grandes almacenes) y ASEDAS (patronal de supermercados). Según este convenio, estas patronales se comprometen a difundir las campañas divulgativas del MSC y a desarrollar actos informativos dirigidos a los padres un viernes y un sábado, al menos 2 veces al año. Durante estos actos, las cajeras distribuirán folletos informativos y los establecimientos exhibirán carteles del ministerio.

Paralelamente, los días 14 y 15 de junio se celebró en Madrid una Conferencia de Prevención y Promoción de la Salud en la Práctica Clínica en España, uno de cuyos 4 temas era el alcohol, donde expertos de diversas procedencias consensuaron unos criterios de prevención basados en la evidencia científica, que aportaron en aras a promover la salud de los ciudadanos, que es el único interés que mueve a los sanitarios.

Aunque las políticas nacionales son competencia de los Estados miembros, la Comisión Europea fomenta la cooperación y coordinación entre ellos, prestará apoyo a las acciones de cada Estado y concederá especial prioridad a todas aquellas que contribuyan a evitar el consumo en los menores y a reducir el daño relacionado con el alcohol, especialmente en menores y jóvenes, así como en el neonato, de acuerdo con las prioridades de la Estrategia Europea ${ }^{3}$.

A partir del 1 de julio de 2007, ya no pueden hacerse alegaciones sobre las virtudes saludables de las bebidas alcohólicas de graduación superior a 1,2\% (por ej., el vino), dado que así queda recogido en el Reglamento Europeo y del Consejo, de 20 de diciembre de 2006, relativo a las declaraciones nutricionales y de propiedades saludables de los alimentos ${ }^{11}$

Paralelamente, la CE recomienda la inclusión de advertencias sanitarias en los envases de todas las bebidas con graduación alcohólica superior a 1,5\% .

También aquí, la Directiva europea Televisión sin Fronteras (89/552/EEC), cuya revisión fue aprobada el 24 de mayo de 2007 (Directiva 97/36/EC), nos aporta algún avance, a pesar de no haber cubierto ni mucho menos las expectativas en ella depositadas.

En todos los países, y a nivel comunitario europeo, existe una fuerte oposición por parte de las partes interesadas a cualquier legislación que amenace con reducir el consumo de alcohol (de adultos o de adolescentes, todo suma) y ello explica que se sucedan las batallas entre economía y salud a todos los niveles. A pesar de ello, en Europa se han ido dando pasos, pequeños pero significativos, en los últimos años. Por primera vez existe una Estrategia sobre alcohol europea $^{3}$ menos ambiciosa que la propuesta, pero un paso trascendental en el lento proceso de concienciar a la población y a sus dirigentes de que las bebidas alcohólicas no son una 'mercancía ordinaria'6.

Por tanto, la coyuntura europea es favorable a una legislación sobre alcohol, dando prioridad a la protección de los menores. De una u otra forma, España debe subirse a este carro y ningún interés económico o político excusaría una inhibición en este sentido. 


\section{REFERENCIAS}

1 Observatorio Español sobre Drogas, Informe 2004. Madrid. Ministerio de Sanidad y Consumo. Delegación del Gobierno para el Plan Nacional sobre Drogas, 2005

2 Rodríguez-Martos A. Ley del Botellón versus Ley de la Bota de Vino. Adicciones 2003; 15: 3-5.

3 Comunicación de la Comisión al Consejo, al Parlamento Europeo, al Comité Económico y Social Europeo y al Comité de las Regiones. Una estrategia de la Unión Europea para ayudar a los Estados miembros a reducir los daños relacionados con el alcohol. Bruselas, 24.10.2006 COM (2006) 625 final.

4 Calafat A. El abuso de alcohol en los jóvenes. Adicciones 2007; 19: 217-24.

5 Asociación de Usuarios de la Comunicación. Menores y Medios de Comunicación. Publicidad, televisión, Internet. (Documentos AUC, http:// www.auc.es).

6 Babor T, Caetano R, Casswell S, Edwards G, Giesbrecht N, Graham K, Grube J, et al. Alcohol: no ordinary commodity. Oxford. Oxford University Press, 2003.
7 Greenberg MD, Morral AR, Jain AK. How can repeat drunk drivers be influenced to change? Analysis of the association between drunk driving and DUI recidivists'attitudes and beliefs. J Stud Alcohol 2004; 65: 460-63.

8 Conclusiones del Consejo de 5 de junio de 2001 sobre una estrategia comunitaria para reducir los daños derivados del consumo de alcohol - DO C 175 de 20.6.2001, p.1.

9 Recomendación 2001 /458/ce DEL Consejo - DO L 161 de 16.6.2001, p. 38.

10 Asociación de Usuarios de la Comunicación. Nota de prensa, 12-06-07 (http:// www.auc.es).

11 Reglamento (CE) No 1924/2006 del Parlamento Europeo y del Consejo de 20 de diciembre de 2006 relativo a las declaraciones nutricionales y de propiedades saludables en los alimentos. Diario Oficial de la Unión Europea 30.12.2006. L 404/9 - L 404/25. 
